\title{
Meta-análise para estimativas de herdabilidade de caracteres vegetativos e reprodutivos de Coffea arabica $\mathrm{L}$.
}

\section{Meta-analysis for heritability estimates of vegetative and reproductive traits of Coffea arabica $\mathrm{L}$.}

\author{
Samuel Pereira de Carvalho ${ }^{1}$; Telde Natel Custódio; \\ Danielle Pereira Baliza ${ }^{3 *}$; Tiago Teruel Rezende ${ }^{4}$
}

\begin{abstract}
Resumo
A compilação de informações advindas de estudos independentes tem sido dificultada em quase todos os campos da ciência, devido principalmente, ao grande número de trabalhos científicos publicados nos últimos anos. Assim, sumarizar informações tornou-se uma necessidade. Neste contexto, uma metaanálise foi conduzida com o objetivo de sistematizar as estimativas para a herdabilidade de caracteres vegetativos e reprodutivos de cafeeiros (Coffea arabica L.). Foram avaliadas as estimativas de herdabilidade referentes aos seguintes caracteres: altura média da planta, diâmetro médio da copa, vigor vegetativo, produção de café beneficiado, rendimento e resistência a ferrugem. Os dados referentes às estimativas de herdabilidade são provenientes de artigos científicos publicados em revistas nacionais e internacionais, anais de congressos, teses e dissertações. A técnica da meta-análise sistematizou as estimativas da herdabilidade dos diferentes estudos e possibilitou concluir que todos os caracteres avaliados são altamente herdáveis, refletindo a grande variedade genética dos cafeeiros, e possibilitando com que ganhos genéticos satisfatórios possam ser atingidos em programas de melhoramento em que esses caracteres são avaliados.

Palavras-chave: Estimativas combinadas, parâmetros genéticos, variabilidade
\end{abstract}

\begin{abstract}
The compilation of informations resulting from independent studies has been difficulted in almost all scientific fields, mainly due to the great number of scientific papers published in recent years. As a result, summarizing information became a need. In this context, a meta-analysis was conducted with the objective of summarizing the estimates for the heritability for vegetative and reproductive traits of coffee (Coffea arabica L.). Heritability estimates were appraised regarding the following traits: average height of plant, average diameter of the canopy, vegetative vigor, production of processed coffee, yield and rust. The data regarding the heritability estimates are from scientific articles published in national and international journals, congress annals, $\mathrm{PhD}$ thesis and Master dissertations. The technique of metaanalysis summarized the estimates heritability from different studies and made possible to conclude that all of the appraised traits are highly inherited, reflecting the great genetic variety of coffee plants, and that is possible to reach satisfactory genetic gains in improvement programs in which those traits are evaluated.
\end{abstract}

Key words: Combined estimates, genetic parameters, variability

${ }^{1}$ Eng $^{\circ}$ Agr $^{\circ}$, Prof. Dr. da Universidade Federal de Lavras, UFLA, Lavras, MG. E-mail: samuelpc@dag.ufla.br

${ }^{2} \mathrm{Eng}^{\circ} \mathrm{Agr}^{\circ}$, Prof. Dr. da Universidade Federal de São João Del Rei, UFSJ, Campus Alto Paraopeba, Ouro Branco, MG. E-mail: natel@ufsj.edu.br

${ }^{3}$ Eng $^{\mathrm{a}} \mathrm{Agr}^{\mathrm{a}}$, Prof $^{\mathrm{a}} \operatorname{Dr}^{\mathrm{a}}$ do Instituto Federal de Educação, Ciência e Tecnologia do Sudeste de Minas Gerais, Campus Rio Pomba, MG. E-mail: danielle.baliza@ifsudestemg.edu.br

${ }^{4}$ Eng $^{\circ}$ Agro $^{\circ}$, Mestrando em Agronomia/Fitotecnia da UFLA, Lavras, MG. E-mail: tiago.teruel@yahoo.com.br

${ }^{*}$ Autora para correspondência 


\section{Introdução}

Tanto em estatística quanto em genética, são estudadas características em indivíduos sem descontinuidades naturais, chamadas de "variação" ou "caracteres métricos". O estudo de um caráter métrico centraliza-se em torno da análise de sua variação, porque é em termos da variância que são formuladas as questões primárias da genética. $\mathrm{O}$ valor observado quando um caráter é medido no indivíduo é o valor fenotípico desse indivíduo; todas as observações de médias, variâncias e covariâncias devem, claramente, ser baseadas na medida do valor fenotípico (PEREIRA NETO, 1994).

Ramalho, Santos e Pinto (2000), afirmam que um dos parâmetros genéticos de maior utilidade para os melhoristas é a estimativa da herdabilidade $\left(\mathrm{h}^{2}\right)$. Segundo esses autores, a proporção herdável da variabilidade total observada é designada de herdabilidade. A herdabilidade permite antever a possibilidade de sucesso com a seleção, uma vez que reflete a proporção da variação fenotípica que pode ser herdada. Em outras palavras, ela mede a confiabilidade do valor fenotípico como indicador do valor reprodutivo (RAMALHO; SANTOS; PINTO, 2000).

Atualmente, existe um grande número de trabalhos científicos contendo estimativas de herdabilidade para os diferentes caracteres vegetativos e reprodutivos dos cafeeiros (Coffea arabica L.). No entanto, em alguns estudos, para um mesmo caráter, observa-se variação nos valores das estimativas obtidas. Miranda, Perecin e Pereira (2005), avaliando a divergência genética entre progênies $\mathrm{F}_{5}$ de Catuaí Amarelo com o Híbrido de Timor, em Machado, MG, verificaram para altura de plantas que a herdabilidade estimada entre as médias das progênies foi de 0,28. Já Severino (2000), caracterizando progênies de Catimor para a mesma variável encontrou valores de herdabilidade de 0,95 , aproximadamente mais que o dobro dos valores encontrados por Miranda, Perecin e Pereira (2005). Bonomo et al. (2004), em Patrocínio, MG, estudando progênies obtidas de cruzamentos de descendentes do Híbrido de Timor com as cultivares Catuaí Vermelho e Catuaí Amarelo verificaram para o caráter produção de grãos beneficiados que o coeficiente de herdabilidade, estimado com base nas médias das progênies ficou entre 0,70 e 0,80 para as colheitas de 1998 e 1999, enquanto na primeira (1997) e quarta colheitas (2000), o coeficiente de herdabilidade foi de 0,25 e 0,43 respectivamente. Carvalho et al. (2009) estudando o comportamento de 29 progênies $\mathrm{F}_{4}$ resultantes do cruzamento de "Icatu" com "Catimor", no município de Campos Altos, MG, constataram para o mesmo caráter valores de herdabilidade de 0,22.

Esta variação ocorre em função das condições ambientais em que as estimativas foram obtidas, da variabilidade genética presente nos materiais utilizados em cada caso e também do método utilizado para a obtenção da estimativa (RAMALHO; SANTOS; ZIMMERMANN, 1993). No entanto, para determinadas características essa variação dificulta a tomada de decisão de alguns pesquisadores sobre o assunto. Uma maneira para contornar tal problema consiste na obtenção de uma média a partir da combinação das estimativas dos resultados já existentes (KOOTS et al., 1994). As estimativas combinadas podem ser obtidas por meio da meta-análise (GIANNOTTI et al., 2005a).

A meta-análise pode ser definida como um procedimento estatístico que consiste de uma revisão quantitativa e resumida de resultados de estudos distintos, mas relacionados (GIANNOTTI et al., 2005a; (GIANNOTTI; PACKER; MERCADANTE, 2005b). Os métodos estatísticos empregados na meta-análise asseguram a obtenção de uma estimativa combinada e precisa, sobretudo em virtude do aumento do número de observações e, consequentemente, do poder estatístico e da possibilidade de examinar a variabilidade entre os estudos, o que não é possível quando se realiza apenas uma média dos resultados publicados (FAGARD; STAESSEN; THIJS, 1996). Este termo foi introduzido por Glass (1976), como "a análise 
das análises" ou "a análise estatística de uma grande coleção de resultados de estudos individuais, com o propósito de completar as descobertas". No entanto, a técnica da meta-análise tem que ser utilizada com critério, principalmente no que diz respeito à seleção dos dados, pois se a mesma não for bem feita os resultados obtidos ficarão comprometidos.

A técnica de meta-análise foi utilizada pela primeiravezempesquisa educacional, teveumgrande avanço na área de medicina e o seu emprego tem se estendido a várias áreas científicas (LOVATTO et al., 2007). No domínio agrícola, o número de metaanálises publicadas tem aumentado nos últimos anos (GIANNOTTI; PACKER; MERCADANTE, 2002; LOVATTO; SAUVANT, 2002; MARTIN; SAUVANT, 2002; OFFNER; BACH; SAUVANT, 2003; EUGÈNE; ARCHIMÈDE.; SAUVANT, 2004; GIANNOTTI et al., 2005a; GIANNOTTI; PACKER; MERCADANTE, 2005b; HAUPTLI; HAUSCHILD; LOVATTO, 2007; BERGMANN; GONZÁLEZ; ZARBIN, 2009) sinalizando que esse procedimento possa se tornar rotina nesse campo da ciência. Contudo, para a área de grandes culturas, em especial para a cultura do café, não foram encontrados trabalhos envolvendo metaanálise. Assim, objetivou-se com este trabalho, obter estimativas combinadas para herdabilidade de caracteres vegetativos e reprodutivos do café arábica, por meio da técnica de meta-análise.

\section{Material e Métodos}

No presente estudo os dados das estimativas de herdabilidade são referentes aos seguintes caracteres vegetativos e reprodutivos do cafeeiro arábica:

- Altura média da planta;

- Diâmetro médio da copa;

- Vigor vegetativo;

- Produção de café beneficiado;

- Rendimento (peso café cereja/peso café beneficiado);

- Resistência à ferrugem.
Os dados referentes às estimativas de herdabilidade são provenientes de artigos científicos publicados em revistas nacionais e internacionais, anais de congressos, teses e dissertações.

A obtenção das estimativas de herdabilidade combinada através da técnica de meta-análise envolveu as seguintes etapas: análise exploratória do conjunto de estimativas de herdabilidade, verificação das pressuposições estatísticas requeridas, aplicação do teste de homogeneidade entre as estimativas de herdabilidade, obtenção das estimativas de herdabilidade combinada.

A análise exploratória do conjunto de estimativas de herdabilidade foi feita com o objetivo de verificar a presença de "outliers", através do gráfico de "BoxPlot". Nesse dispositivo, observou-se a distribuição e o resumo das principais estatísticas do conjunto de dados, visando, principalmente, verificar se os conjuntos de dados são comparáveis entre si (BUSSAB; MORETTIN, 2003).

As pressuposições estatísticas requeridas para uma meta-análise são a normalidade e a independência das estimativas a serem combinadas (HEDGES; OLKIN, 1985). A suposição de independência é em parte satisfeita, em razão das estimativas terem sido obtidas de trabalhos distintos. A suposição de normalidade foi testada através do teste de Shapiro-Wilk (SHAPIRO; WILK, 1965).

O teste de homogeneidade é importante na decisão de qual modelo deve ser adotado, se de efeito fixo ou aleatório. O modelo de efeito fixo pressupõe homogeneidade entre as estimativas de herdabilidade, ao passo que o modelo de efeito aleatório considera a variabilidade existente entre as estimativas.

O modelo de efeito aleatório é dado por:

$$
\hat{\mathrm{h}}_{\mathrm{i}}^{2}=\hat{\mathrm{h}}_{+}^{2}+\varepsilon_{\mathrm{i}}+\mathrm{e}_{\mathrm{i}},
$$

sendo:

$\hat{\mathrm{h}}_{\mathrm{i}}^{2}$ : valor estimado da i-ésima herdabilidade para determinado caráter; 
$\mathrm{h}_{+}^{2}$ : valor da estimativa combinada das herdabilidades;

$\varepsilon_{\mathrm{i}}$ : erro aleatório, tal que: $\varepsilon_{\mathrm{i}} \stackrel{\text { i.i.d. }}{\sim} \mathrm{N}\left(0, \tau^{2}\right)$;

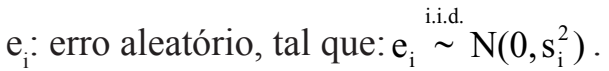

O modelo de efeito fixo é um caso particular do modelo de efeito aleatório em que $\tau^{2}=0$ (BROCKWELL; GORDON, 2001).

O teste de homogeneidade entre as estimativas de herdabilidade foi feito com base na estatística Q (HEDGES; OLKIN, 1985), dada por:

$$
\mathrm{Q}=\sum_{\mathrm{i}=1}^{\mathrm{k}} \mathrm{w}_{\mathrm{i}}\left(\hat{\mathrm{h}}_{\mathrm{i}}^{2}-\hat{\mathrm{h}}_{+}^{2}\right)^{2} \sim \chi_{\mathrm{k}-1}^{2},
$$

sendo:

$\mathrm{w}_{\mathrm{i}}=\frac{1}{\mathrm{~s}_{\mathrm{i}}^{2}} ;$

$\hat{\mathrm{h}}_{\mathrm{i}}^{2}$ : valor estimado da i-ésima herdabilidade para determinado caractere;

$\hat{\mathrm{h}}_{+}^{2}=\frac{\sum_{\mathrm{i}=1}^{\mathrm{k}} \mathrm{w}_{\mathrm{i}} \hat{\mathrm{h}}_{\mathrm{i}}^{2}}{\sum_{\mathrm{i}=1}^{\mathrm{k}} \mathrm{w}_{\mathrm{i}}}$ : valor da estimativa combinada das

$\mathrm{k}=$ número de valores amostrados para o referido caracter;

A estatística Q tem distribuição aproximada de Qui-quadrado com k-1 graus de liberdade.

$\mathrm{s}_{\mathrm{i}}^{2}$ : variância associada a $\hat{\mathrm{h}}_{\mathrm{i}}^{2}$.

As variâncias $\left(s_{i}^{2}\right)$ associadas a $\hat{\mathrm{h}}_{\mathrm{i}}^{2}$ foram estimadas pelo método descrito por Falconer e Mackay (1996), dada por:

$$
\hat{\mathrm{V}}\left(\hat{\mathrm{h}}_{\mathrm{i}}^{2}\right)=\frac{32 \hat{\mathrm{h}}_{\mathrm{i}}^{2}}{\mathrm{~N}} .
$$

O desvio padrão combinado $\left(\mathrm{s}_{+}\right)$associado a $\hat{\mathrm{h}}_{+}^{2}$, foi estimado (KOOTS et al., 1994) por:

$$
\mathrm{s}_{+}=\sqrt{\frac{1}{\sum_{\mathrm{i}=1}^{\mathrm{k}}\left(\frac{1}{\mathrm{w}_{\mathrm{i}}}\right)}} .
$$

As análises foram feitas através de rotinas computacionais do programa SAS (ARTHUR JUNIOR, BENNETT JUNIOR; HUFFCUTT, 2008).

\section{Resultados e Discussão}

$\mathrm{Na}$ Tabela 1 são apresentados os valores mínimos, máximos e amplitudes de variação das estimativas de herdabilidade para os caracteres avaliados, na qual pode-se verificar, pelos valores apresentados, que todos os caracteres avaliados apresentaram uma amplitude de variação bastante alta para as estimativas de herdabilidade, ou seja, resultados discordantes quanto aos valores encontrados na literatura, justificando assim, a aplicação da técnica de meta-análise. Amplitudes menores podem sinalizar uma melhor qualidade dos estudos em decorrência de uma possível melhoria desses métodos de estimação dos parâmetros genéticos e da qualidade e quantidade dos bancos de dados envolvidos (GIANNOTTI; PACKER; MERCADANTE, 2002).

Para a análise exploratória, construíram-se gráficos de "box-plot" para os caracteres em estudo. Nesse dispositivo, observou-se a distribuição e o resumo das principais estatísticas do conjunto de dados (mediana, quartis e prováveis dados discrepantes) (BUSSAB; MORETTIN, 2003). Para o conjunto das estimativas de herdabilidade de todos os caracteres avaliadas houve ausência de "outliers", isto é, não ocorreram observações discrepantes entre as características em estudo (Figura 1). 
Tabela 1. Valores mínimo, máximo, amplitude, média geral, coeficiente de variação e número de estimativas $(\mathrm{N})$ de herdabilidades amostradas dos caracteres avaliados.

\begin{tabular}{ccccccc}
\hline & \multicolumn{7}{c}{ Caracteres } \\
\cline { 2 - 7 } Herdabilidade & Altura & Ferrugem & Rendimento & Vigor & Diâmetro da copa & Produção \\
\hline Mínimo & 0,21 & 0,67 & 0,49 & 0,30 & 0,28 & 0,24 \\
Máximo & 0,95 & 0,96 & 0,85 & 0,89 & 0,95 & 0,93 \\
Amplitude & 0,74 & 0,29 & 0,36 & 0,59 & 0,67 & 0,68 \\
$\mathrm{~N}$ & 15 & 16 & 18 & 19 & 12 & 123 \\
\hline Média aritmética & 0,65 & 0,84 & 0,69 & 0,65 & 0,66 & 0,66 \\
C.V. (\%) & 38,88 & 11,80 & 15,93 & 31,42 & 36,33 & 25,81 \\
\hline
\end{tabular}

Fonte: Elaboração dos autores.

Figura 1. Box-Plot das estimativas de herdabilidade para os caracteres avaliados.

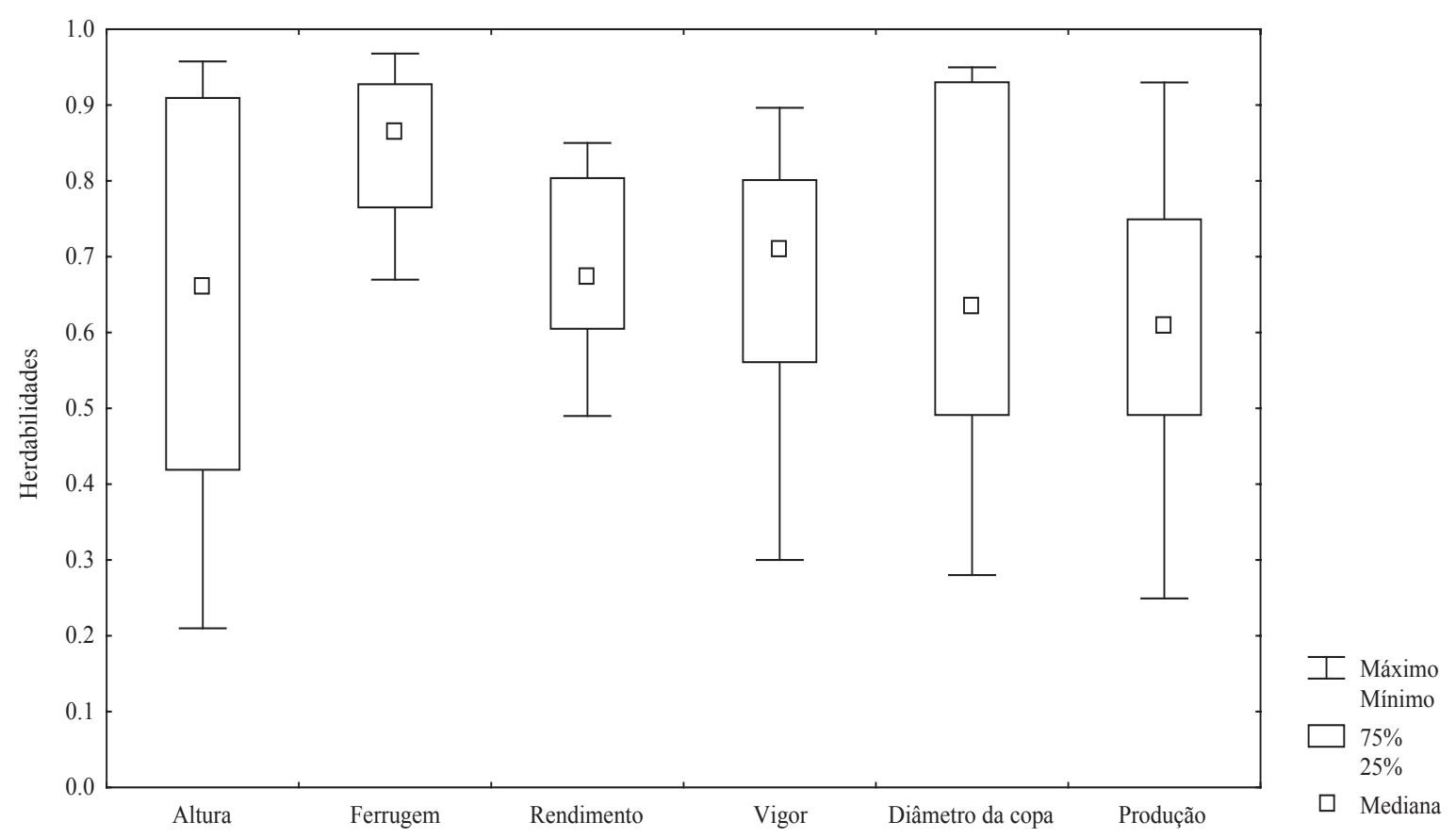

Fonte: Elaboração dos autores.

Assim, procedeu-se a meta-análise, verificando as pressuposições de normalidade e homogeneidade requeridas para a aplicação desta técnica. A suposição de normalidade foi testada através do teste de Shapiro-Wilk (SHAPIRO; WILK, 1965) e os resultados do teste mostraram que todas as características avaliadas seguem uma distribuição de probabilidades normal (Tabela 2). O prosseguimento da meta-análise se deu com a aplicação do teste de homogeneidade entre as estimativas de herdabilidade com base na estatística Q, cujos resultados estão apresentados na Tabela 2. 
Tabela 2. Valores das estatísticas do teste de ShapiroWilk (W) para normalidade e do teste de homogeneidade (Q) e os respectivos p-valor entre as estimativas de herdabilidade, para os caracteres avaliados.

\begin{tabular}{lcccc}
\hline \multicolumn{1}{c}{ Caracteres } & W & p-valor & Q & p-valor \\
\hline Altura & 0,91 & 0,16 & 2,74 & 0,99 \\
Ferrugem & 0,93 & 0,38 & 0,30 & 1,00 \\
Rendimento & 0,93 & 0,34 & 0,42 & 1,00 \\
Vigor & 0,89 & 0,10 & 1,98 & 0,99 \\
Diâmetro da copa & 0,92 & 0,36 & 1,27 & 0,99 \\
Produção & 0,97 & 0,07 & 7,18 & 1,00 \\
\hline
\end{tabular}

Fonte: Elaboração dos autores.

O teste de homogeneidade é importante na decisão de qual modelo deve ser adotado, se de efeito fixo ou aleatório. O modelo de efeito fixo pressupõe homogeneidade entre as estimativas de herdabilidade, ao passo que o modelo de efeito aleatório considera a variabilidade existente entre as estimativas. Na Tabela 2 observa-se por meio dos valores ( $\mathrm{p}$-valor) que a hipótese de homogeneidade entre as estimativas de herdabilidade para todos os caracteres avaliados não deve ser rejeitada. Logo, adotou-se um modelo de efeito fixo. Esses resultados indicam que no teste de homogeneidade não existem diferenças substanciais entre os trabalhos científicos analisados neste estudo.
Giannotti et al. (2005a) e Giannotti, Packer e Mercadante (2005b), realizaram meta-análise em estimativas de herdabilidade para características de crescimento em bovinos de corte. Após verificarem ausência de homogeneidade adotaram o modelo de efeito aleatório. Essa diferença entre os modelos adotados (fixo ou aleatório) em trabalhos efetuados no domínio agrícola, talvez possa ser explicado pelo fato dos trabalhos de Giannotti et al. (2005a) e Giannotti, Packer e Mercadante (2005b) terem sido realizados com animais e o presente trabalho ser feito com espécie vegetal. No entanto, mais trabalhos com espécies vegetais devem ser realizados para comparações de resultados.

Prosseguiu-se com o processo de obtenção da estimativa combinada das herdabilidades $\left(\hat{\mathrm{h}}_{+}^{2}\right)$ para os caracteres avaliados, seu desvio padrão combinado e o coeficiente de variação associado a $\hat{h}_{+}^{2}$, cujos valores encontram-se na Tabela 3, bem como aqueles obtidos pelo método convencional. Para todos os caracteres avaliados foram encontrados altos valores para as estimativas combinadas de herdabilidade, refletindo a grande variedade genética dos cafeeiros, além de possibilitar que ganhos genéticos satisfatórios possam ser atingidos em programas de melhoramento em que essas características são avaliadas.

Tabela 3. Estimativas convencionais e combinadas das herdabilidades, seus desvios padrões e respectivos coeficientes de variação, para os caracteres avaliados.

\begin{tabular}{lccccccc}
\hline & \multicolumn{2}{c}{$\begin{array}{c}\text { Estimativas convencionais } \\
\text { (Médias aritméticas) }\end{array}$} & & \multicolumn{3}{c}{$\begin{array}{c}\text { Estimativas combinadas } \\
\text { (Meta- análise) }\end{array}$} \\
\cline { 2 - 3 } \cline { 6 - 8 } \multicolumn{1}{c}{ Caracteres } & Herdabilidade & Desvio padrão & $\mathrm{Cv}(\%)$ & & $\hat{\mathrm{h}}_{+}^{2}$ & $\mathrm{~s}_{+}$ & $\mathrm{Cv}(\%)$ \\
\hline Altura & 0,65 & 0,25 & 38,46 & & 0,57 & 0,10 & 17,54 \\
Ferrugem & 0,84 & 0,09 & 10,71 & & 0,82 & 0,11 & 13,42 \\
Rendimento & 0,69 & 0,11 & 15,94 & & 0,68 & 0,11 & 16,18 \\
Vigor & 0,65 & 0,20 & 30,77 & & 0,51 & 0,11 & 21,57 \\
Diâm. copa & 0,66 & 0,24 & 36,36 & & 0,61 & 0,15 & 24,59 \\
Produção & 0,66 & 0,17 & 25,76 & & 0,54 & 0,02 & 3,70 \\
\hline
\end{tabular}

Fonte: Elaboração dos autores. 
A magnitude das herdabilidades é muito importante em programas de melhoramento, pois, se uma característica é altamente herdável, pode-se afirmar que pais com alto desempenho tenderão a produzir progênies também com alto desempenho (RAMALHO; SANTOS; PINTO, 2008; BORÉM, 2001), ou seja, quanto maior o valor estimado da herdabilidade maior será a chance de sucesso com a seleção (VASCONCELOS et al., 2012). Conforme relatado por Bourdon (2000), são consideradas pouco herdáveis estimativas de herdabilidades com valores abaixo de 0,20, moderadamente herdáveis estimativas com valores entre 0,20 e 0,40 e altamente herdáveis estimativas com valores superiores a 0,40 .

A maior estimativa combinada de herdabilidade foi encontrada para ferrugem, refletindo a grande variabilidade genética de cafeeiros e a baixa influência ambiental, o que permite que ganhos genéticos satisfatórios possam ser atingidos em programas de melhoramento que inclua este caráter em seu critério de seleção.

Aparentemente, caracteres com elevadas estimativas de herdabilidade, pouco diferem na magnitude em função do método de obtenção utilizado, quer por simples média aritmética, que por meta-analise, como foi o caso da reação à ferrugem, com estimativa de cerca de 0,85. No entanto, em variáveis com maior influência ambiental, a estimativa obtida por meta-análise mostra-se mais conservadora (de menor magnitude), porém com maior confiabilidade (menor desvio), como o demonstra o caracter produção de grãos (Tabela 3).

De acordo com Fagard, Staessen e Thijs (1996) os métodos estatísticos empregados na meta-análise asseguram a obtenção de uma estimativa combinada e precisa, sobretudo em virtude do aumento do número de observações e, consequentemente, do poder estatístico e da possibilidade de examinar a variabilidade entre os estudos, o que não é possível quando se realiza apenas uma média dos resultados publicados. Dessa forma, as estimativas combinadas das herdabilidades encontradas neste estudo podem contribuir muito para o avanço do melhoramento genético do cafeeiro, servindo de referências para futuras consultas dos valores de herdabilidade, principalmente para os futuros trabalhos de melhoramento genético de Coffea arabica.

\section{Conclusões}

A meta-análise indica que o componente genético é preponderante frente ao ambiental, na variabilidade fenotípica dos caracteres vegetativos e reprodutivos de cafeeiros da espécie Coffea arabica $\mathrm{L}$.

\section{Agradecimentos}

Ao Conselho Nacional de Desenvolvimento Científico e Tecnológico (CNPq) pelo apoio financeiro.

\section{Referências}

ARTHUR JUNIOR, W.; BENNETT JUNIOR, W.; HUFFCUTT, A. I. Conducting meta-analysis using SAS. 2. ed. New Jersey: Psychonology Press, 2008. 188 p.

BERGMANN, J.; GONZÁLEZ, A.; ZARBIN, P. H. G. Insect pheromone research in South America. Journal of Brazilian Chemical Society, São Paulo, v. 20, n. 7, p. 1206-1219, 2009.

BONOMO, P.; CRUZ, C. D.; VIANA, J. M. S.; PEREIRA, A. A.; OLIVEIRA, V. R.; CARNEIRO, P. C. S. Avaliação de progênies obtidas de cruzamentos de descendentes do Híbrido de Timor com as cultivares Catuaí Vermelho e Catuaí Amarelo. Bragantia, Campinas, v. 63, n. 2, p. 207-219, 2004.

BORÉM, A. Melhoramento de plantas. 3. ed. Viçosa: UFV. 2001. 500 p.

BOURDON, G. E. P. Understanding animal breeding. Upper Saddle River: Prendice-Hall, 2000. 538 p.

BROCKWELL, S. E.; GORDON, I. R. A comparation of statistical methods for meta-analysis. Statistics in Medicine, Chichester, v. 20, n. 6, p. 825-840, 2001.

BUSSAB, W. O.; MORETTIN, P. A. Estatística básica. 5. ed. São Paulo: Saraiva, 2003. 526 p. 
CARVAlHO, G. R.; BOTELHO, C. E.; BARTHOLO, G. F.; PEREIRA, A. A.; NOGUEIRA, A. M.; CARVALHO, A. M. Comportamento de progênies $\mathrm{F}_{4}$ obtidas por cruzamentos de 'Icatu' com 'Catimor'. Ciência e Agrotecnologia, Lavras, v. 33, n. 1, p. 47-52, 2009.

EUGÈNE, M.; ARCHIMÈDE, H.; SAUVANT, D. Quantitative meta-analysis on the effects of defaunation of the rumen on growth, intake and digestion in ruminants. Livestock Production Science, Amsterdam, v. 85, n. 1, p. 81-97, 2004.

FAGARD, R. H.; STAESSEN, J. A.; THIJS, L. Advantages and disadvantages of the meta-analysis approach. Journal of Hypertension, v. 14, p. 9-13, 1996. Supplement 2.

FALCONER D. S.; MACKAY, T. F. C. Introduction to quantitative genetics. Edinburgh: Addison Wesley Longman, 1996. 464 p.

GIANNOTTI, J. D. G.; PACKER, I. U.; MERCADANTE, M. E. Z. Meta-análise para estimativas de herdabilidade para características de crescimento em bovinos de corte. Revista Brasileira de Zootecnia, Viçosa, MG, v. 34, n. 4, p. 1173-1180, 2005b.

- Meta-análise para estimativas de correlação genética entre pesos ao nascer e desmama de bovinos. Scientia Agricola, Piracicaba, v. 59, n. 3, p. 435-440, 2002.

GIANNOTTI, J. D. G.; PACKER, I. U.; MERCADANTE, M. E. Z.; LIMA, C. G. de. Análise de agrupamento para implementação da meta-análise em estimativas de herdabilidade para características de crescimento em bovinos de corte. Revista Brasileira de Zootecnia, Viçosa, MG, v. 34, n. 4, p. 1165-1172, 2005 a.

GLASS, G. V. Primary, secondary, and meta-analysis of research. Educational Researcher, Washington, v. 5, n. 10, p. 3-8, 1976.

HAUPTLI, L.; HAUSCHILD, L.; LOVATTO, P. A. Adição de extratos vegetais e antimicrobianos de síntese para leitões na creche: Estudo meta-analítico. Ciência Rural, Santa Maria, v. 7, in press, 2007.

HEDGES, L. V.; OLKIN, I. Statistical methods for metaanalysis. London: Academic Press, 1985. 369 p.

KOOTS, K. R.; GIBSON, J. P.; SMITH, C.; WILTON, J.W. Analysis of published genetic parameter estimates for beef production traits. 1. Heritability. Animal Breeding Abstracts, Wallingford, v. 62, n. 5, p. 309-338, 1994.

LOVATTO, P. A.; LEHNEN, C. R.; ANDRETA, I.; CARVALHO, A. D.; HAUSCHILD, L. Meta-análise em pesquisas científicas - enfoque em metodologias. Revista Brasileira de Zootecnia, Viçosa, MG, v. 36, p. 285-294, 2007. Suplemento.

LOVATTO, P. A.; SAUVANT, D. Méta-analyse et modélisation de l'ingestion volontaire chez le porc en croissance. Journées Rech. Porcine en France, Paris, v. 34, p. 129-134, 2002.

MARTIN, O.; SAUVANT, D. Meta-analysis of input/ output kinetics in lactating dairy cows. Journal Dairy Science, Savoy, v. 85, n. x, p. 3363-3381, 2002.

MIRANDA, J. M.; PERECIN, D.; PEREIRA, A. A. Produtividade e resistência à ferrugem do cafeeiro (Hemileia vastatrix berk. et br.) de progênies $\mathrm{F}_{5}$ de Catuaí Amarelo com o Híbrido de Timor. Ciência e Agrotecnologia, Lavras, v. 29, n. 6, p. 1195-1200, 2005.

OFFNER, A.; BACH, A.; SAUVANT, D. Quantitative review of in situ starch degradation in the rumen. Animal Feed Science and Technology, Amsterdam, v. 106, n. 1-4, p. 81-93, 2003.

PEREIRA NETO, J. Estudo comparativo de métodos de estimação da variância de coeficiente de herdabilidade. 1994. Dissertação (Mestrado em Agronomia. Estatística e Experimentação Agronômica) - Escola Superior de Agricultura Luiz de Queiroz. Universidade de São Paulo, Piracicaba.

RAMAlHO, M. A. P.; SANTOS, J. B.; PINTO, C. A. B. P. Genética na agropecuária. 2. ed. Lavras: UFLA, 2000. 472 p. 4. ed. Lavras: UFLA, 2008. 463 p.

RAMALHO, M.A. P.; SANTOS, J. B.; ZIMMERMANN, M. J. O. Genética quantitativa em plantas autógamas: aplicações ao melhoramento do feijoeiro. Goiânia: UFG, 1993. $271 \mathrm{p}$.

SEVERINO, L. S. Caracterização de progênies de Catimor e avaliação de descritores em Coffea arabica L.. 2000. Tese (Doutorado em Fitotecnia) - Universidade Federal de Viçosa, Viçosa.

SHAPIRO, S. S.; WILK, M. B. An analysis of variance test for normality (complete samples). Biometrika, London, v. 52, n. 3-4, p. 591-611, 1965.

VASCONCELOS, E. S. de; REIS, M. S.; SEDIYAMA, T.; CRUZ, C. D. Estimativas de parâmetros genéticos da qualidade fisiológica de sementes de genótipos de soja produzidas em diferentes regiões de Minas Gerais. Semina: Ciências Agrárias, Londrina, v. 33, n. 1, p. $65-$ $76,2012$. 